\title{
RUNWAY INCURSION PREVENTION FOR GENERAL AVIATION OPERATIONS
}

\author{
Denise R. Jones and Lawrence J. Prinzel, III \\ National Aeronautics and Space Administration, Hampton, VA
}

\begin{abstract}
A Runway Incursion Prevention System (RIPS) and additional incursion detection algorithm were adapted for general aviation operations and evaluated in a simulation study at the National Aeronautics and Space Administration (NASA) Langley Research Center (LaRC) in the fall of 2005. RIPS has been designed to enhance surface situation awareness and provide cockpit alerts of potential runway conflicts in order to prevent runway incidents while also improving operational capability. The purpose of the study was to evaluate the airborne incursion detection algorithms and associated alerting and airport surface display concepts for general aviation operations. This paper gives an overview of the system, simulation study, and test results.
\end{abstract}

\section{Introduction}

Runway incursions are a serious aviation safety hazard, particularly for general aviation (GA) operations. According to the Federal Aviation Administration (FAA) [1], during the four year period from fiscal year (FY) 2001 through FY 2004, there were approximately 257 million aircraft operations and 1,395 runway incursions reported at United States towered airports - approximately 5.4 runway incursions for every one million operations. General aviation accounted for 74 percent of these incursions but only 57 percent of the operations. Seventy-six percent of the most severe incursions (114 of 150 incursions) involved at least one GA aircraft. Five incursions resulted in collisions, with four of these collisions involving GA aircraft. These statistics do not consider incidents that occur at non-towered airports.

Current FAA initiatives are targeting reductions in the severity, number, and rate of runway incursions by implementing a combination of technology, infrastructure, procedural, and training interventions [1]. None of these initiatives involve technology solutions for the aircraft.

The National Transportation Safety Board (NTSB) also considers runway incursions to be a serious aviation safety hazard, listing runway incursion prevention as one of their "most wanted" transportation safety improvements [2]. The NTSB specifically recommends that the FAA implement technology that "provides a direct warning capability to flight crews" [3].

NASA developed a Runway Incursion Prevention System (RIPS) for commercial aviation operations to improve airport safety by providing supplemental surface situation awareness information and guidance cues, and alerts of runway conflicts and route deviations directly to the flight crew. The system was evaluated in several flight tests and simulation studies [4][5][6].

RIPS was adapted for GA operations and integrated with NASA's GA synthetic vision system. A piloted simulation study was conducted at NASA LaRC to evaluate RIPS for GA operations focusing on analysis of the incursion detection algorithms and display concepts. This paper will present an overview of the system, description of the simulation study, and reporting of test results.

\section{System Description}

\section{Simulation Facility \\ Flight Deck Simulator \\ NASA LaRC's Integration Flight Deck (IFD) transport category fixed-based high-fidelity flight simulator was used for this study because of its visual, tactile, and audio capabilities. The IFD was adapted to emulate a Cessna 206 (C-206) GA aircraft (herein referred to as the ownship). A basic six degree of freedom non-linear simulation model}


of the C-206 and representative control force and braking models were used. In order to avoid distractions, extraneous displays and gauges were covered or turned off during data collection. This configuration was used successfully in a previous GA simulation [7].

As shown in Figure 1, an electronic research display (RD) was installed on the instrument panel directly in front of the left seat and control yoke. The RD was composed of two 10.4" liquid crystal displays and was capable of displaying two separate digital displays, side-by-side. An electronic flight bag (EFB) display, located to the left of the RD, was used to present the airport surface map display concepts described below. This display was 10.4" diagonal with a resolution of $1280 \times 1024$ pixels. The collimated out-the-window scene provided a 200 degree horizontal by 40 degree vertical fieldof-view at 26 pixels per degree resolution.

Traffic position data was "broadcast" at a $1 \mathrm{~Hz}$ rate. No additional latency in traffic position was used. Ownship position data was updated at a 20 $\mathrm{Hz}$ rate. Positional error was not introduced into these data.

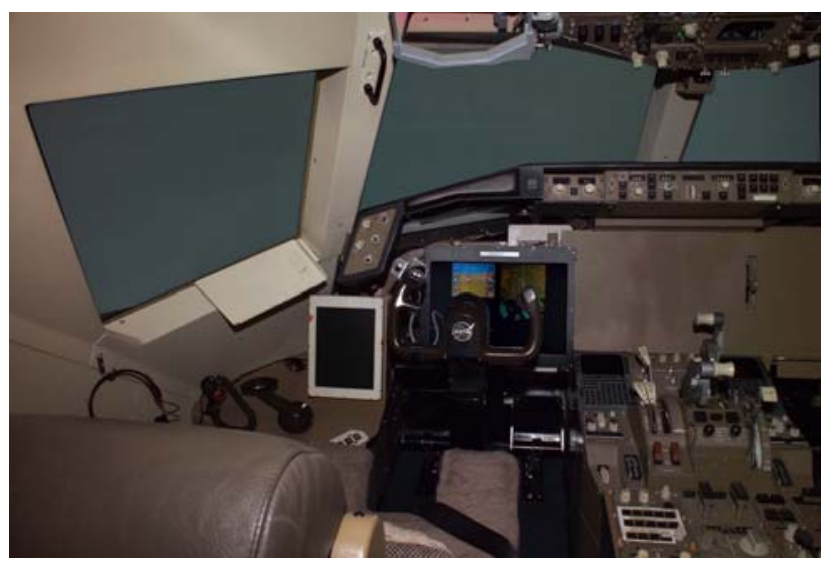

Figure 1. IFD Flight Deck

\section{Air Traffic Control Simulation}

An air traffic control (ATC) simulation was created to establish a realistic terminal area environment. Approach and tower ATC instructions and traffic requests and replies were prerecorded using different voices. The messages were then played through the flight deck speaker system when the ownship and simulated traffic reached specified locations.

\section{Research Displays}

This simulation study was geared for low end GA aircraft; therefore, standard round dial instrumentation was used in conjunction with various airport surface map formats and alerting. The round dial displays were shown on the RD located in front of the evaluation pilot (EP) (see Flight Deck Simulator section). When required, an airport surface map was displayed on the EFB located to the left of the round dial display. The surface map was generated using a Reno International (RNO) airport geographic database developed to RTCA standards [8]. The map scale was set to $2.5 \mathrm{~nm}$ for the airborne scenarios and 1.5 $\mathrm{nm}$ for the ground based scenarios. Audible alerts sounded through the flight deck speaker system.

Seven display conditions were evaluated during the course of the study as described below.

\section{Baseline (B)}

The Baseline (B) display condition consisted of a set of six GA instruments (airspeed, attitude, altitude, vertical speed, directional gyro, and turn coordinator) plus manifold pressure and instrument landing system data. All instruments were three inches in diameter and configured on the $\mathrm{RD}$ as shown in Figure 2.
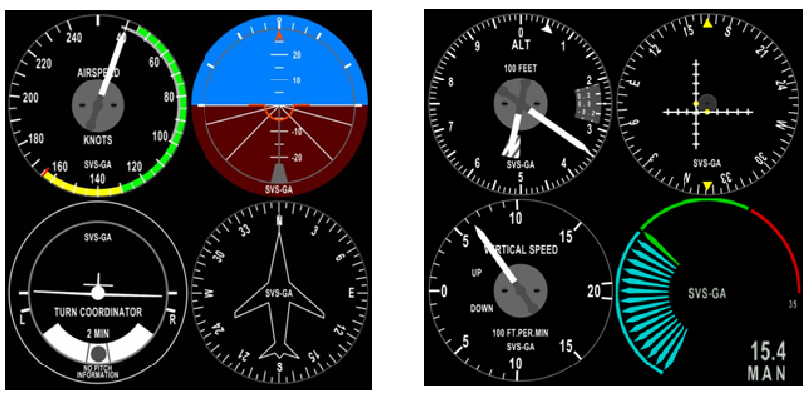

Figure 2. Baseline Display Condition (BMO)

Baseline with Surface Map and Ownship

The BMO display condition consisted of the baseline round dials displayed on the RD with the addition of a plan view surface map displayed on the EFB. This version of the surface map displayed an airport layout along with ownship position. Traffic was not shown. Incursion alerting was not part of this condition. 


\section{Baseline with Surface Map and Ownship and Traffic (BMOT)}

The BMOT display condition was equivalent to the BMO condition with the addition of traffic on the surface map (Figure 3). Traffic was indicated by dark blue chevrons when on the ground and cyan chevrons when airborne. Incursion alerting was not part of this condition.

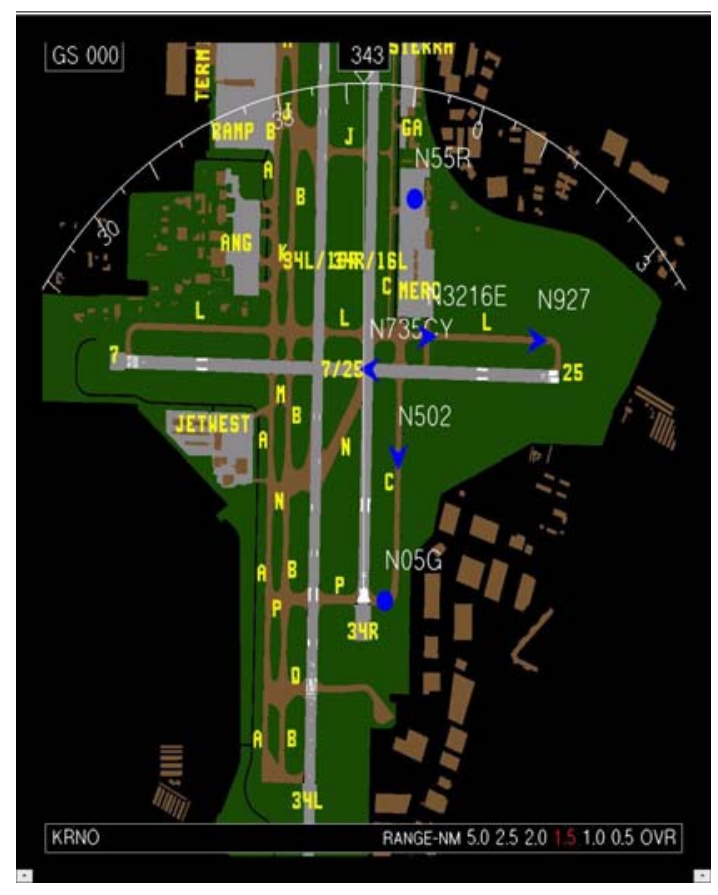

Figure 3. Plan View Surface Map

Baseline with Audible Incursion Alerts (BA)

The BA display condition consisted of the baseline round dials displayed on the RD and audible runway incursion alerts sounded in the flight deck when a potential conflict was detected.

Baseline with Surface Map and Ownship and Audible Incursion Alerts (BAMO)

The BAMO display condition was equivalent to the BMO condition with the addition of audible runway incursion alerts.

Baseline with Surface Map and Ownship, Traffic, and Incursion Alerts (BAMOT)

The BAMOT display condition was equivalent to the BMOT condition with the addition of both audible and graphical runway incursion alerts. The alert implementation is described in detail below in the Runway Incursion Alerting section.

\section{(BRIPS)}

Baseline with Perspective Surface Map

The BRIPS display condition consisted of the baseline round dials displayed on the RD with a perspective surface map displayed on the EFB. The map showed a perspective track-up airport layout with ownship and traffic locations, ATC instructions (including the approved taxi route and hold short locations), and incursion alerts (Figure 4). Audible incursion alerts were also sounded.

Audible route deviation and crossing hold alerts were also generated. Route deviation alerts were generated if ownship left its assigned path during taxi. Crossing hold alerts were generated if ownship crossed a hold line without clearance.

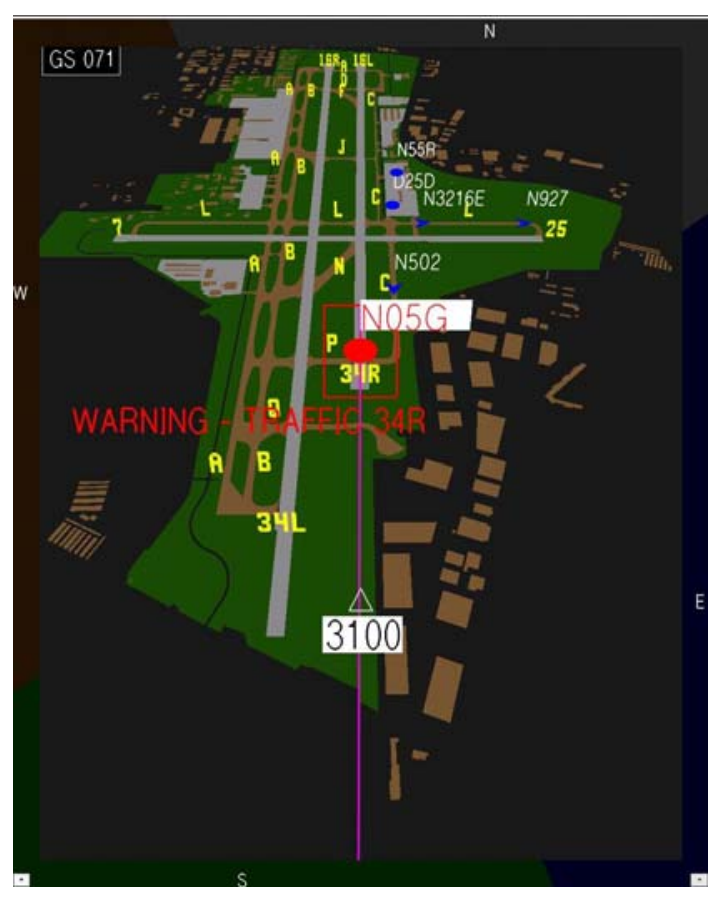

Figure 4. Perspective Surface Map

\section{Runway Incursion Alerting}

The runway was monitored for potential incursions any time the ownship was to enter the runway - during final approach and landing, takeoff roll, and taxi crossing. If an incursion was predicted, audible and graphical alerts were generated and presented to the flight crew. These aircraft-generated alerts could also be data linked to ATC so the pilots and controllers have the same awareness. Currently, conflict resolution advisories are not provided. 
Two different incursion detection algorithms were evaluated during the simulation study.

The Runway Safety Monitor (RSM) incursion detection algorithm [9] uses a generic approach for detecting and generating incursion alerts and is not designed to detect only specific incursion scenarios. The RSM monitors traffic that enters a threedimensional virtual protection zone around the runway that is being used by the ownship. Incursion detection is based on the operational state of the ownship and traffic, as well as other criteria (separation and closure rate), to avoid false alerts. Identification, position, and altitude data is used to track the traffic in the protection zone. Traffic data projections are calculated within RSM since, from flight test experience, reliable position updates are not received at consistent intervals. RSM generates a Warning alert, which occurs when a runway incursion is detected and evasive action is required to avoid a potential collision. Information provided with each alert includes identification of the incurring traffic and separation distance to potential conflict. RSM was developed for NASA by Lockheed Martin.

The PathProx ${ }^{\mathrm{TM}}$ detection algorithm [10] is designed to handle over 40 specific runway incursion scenarios. Alerts are issued based on the states of the ownship and traffic and on conditions including position, speed, and track angle.

PathProx ${ }^{\mathrm{TM}}$ generates two types of alerts analogous to the Traffic Alert and Collision Avoidance System (TCAS) approach. A Caution alert informs the flight crew of a potential incursion or an incursion where the conflict does not yet require evasive action. The crew can take evasive action, however, at their discretion. PathProx ${ }^{\mathrm{TM}}$ also generates Warning alerts when immediate evasive action is required. Information provided with each alert includes identification of the incurring traffic, the associated runway, and separation distance between the traffic and ownship. PathProx ${ }^{\mathrm{TM}}$ was developed by Rannoch Corporation.

For this test, the incursion alerts were presented to the flight crew both visually on the surface map and audibly, if required by the current display condition. The alert phrases were designed to provide descriptive information regarding the location of the incurring traffic, e.g. "Warning, Traffic Departing 25", "Caution, Traffic 34R". The textual forms of these alerts were presented on the surface map. Also, the traffic symbol representing the incurring aircraft was enlarged, changed color (yellow for Caution and red for Warning), and was highlighted by a target designator box. The identification tag was also highlighted. In the event the incurring traffic symbol was not shown because of the display scale, a symbol was pegged on the edge of the display in the direction of the traffic on the perspective surface map only. The distance to conflict was shown beneath the ownship symbol. Figure 4 shows and example of a Warning alert.

\section{Test Method}

Data collection occurred for several different scenarios and test conditions as described below.

\section{Test Scenarios}

Five incursion scenarios were evaluated during the simulation. The EP was trained to abort if a warning alert was given during departure, goaround if a warning alert was given on approach, and stop if a warning alert was given during taxi. The EP was not required to take evasive action when a caution alert was issued.

A traffic pattern was established to emulate a representative GA traffic flow at RNO. Alternating arrivals and departures were on Runway 34R with interleaving departures on Runway 25. Traffic traveled through the intersection of runways 25 and $34 \mathrm{R}$ every minute. The incurring traffic was interleaved into this traffic flow.

Every effort was made to produce similar timing for the scenarios; however, a certain amount of variability was naturally introduced due to the maneuvering conducted by the EP (i.e., approach speed, taxi speed, etc.).

\section{Scenario 1 - arrival/take-off hold}

Scenario 1 tested the incursion situation where an aircraft was in position and holding for departure clearance while another aircraft was approaching the same runway for a landing (Figure 5). The ownship was approaching Runway 34R, $3 \mathrm{~nm}$ from the threshold at 1010' above field level (AFL) and at an indicated airspeed of $90 \mathrm{kts}$. The EP was instructed to land. The traffic was initially at the $34 \mathrm{R}$ hold line nearest to the runway threshold. The 
traffic was cleared to taxi into position on the active runway when the ownship was $2 \mathrm{~nm}$ from the threshold and held in that location awaiting departure clearance.

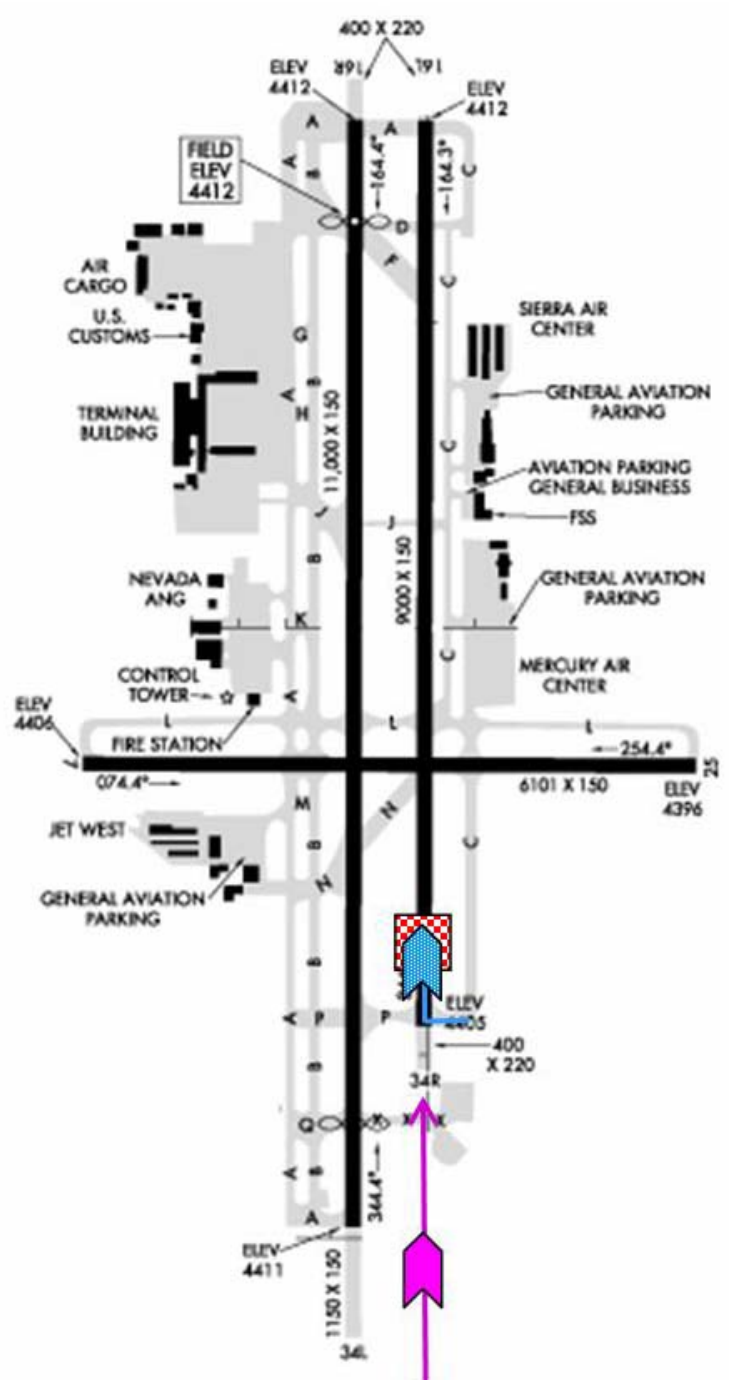

Figure 5. Scenario 1 Configuration

\section{Scenario 2 - departure/intersection departure}

Scenario 2 tested the case where one aircraft was departing as another aircraft entered the runway for an intersection departure. The ownship was initially on Taxiway C approximately 500' from the Runway 34R threshold traveling at $8 \mathrm{kts}$. The traffic was located at the 34R hold line on Taxiway L for an intersection departure. (Taxiway L is approximately 3000 ' from Runway $34 \mathrm{R}$ threshold.) The ownship was cleared for departure.
Once the ownship began its departure (on runway heading and traveling greater than $10 \mathrm{kts}$ ), the traffic taxied across the hold line and entered Runway 34R without clearance.

\section{Scenario 3 - arrival/departure}

Scenario 3 was a crossing runway scenario with one aircraft landing at the same time another aircraft was departing on an intersecting runway. The ownship was approaching Runway 34R, 3nm from the threshold at 1010' AFL and at an indicated airspeed of $90 \mathrm{kts}$. The EP was instructed to land and stop on the runway. The traffic was initially on Taxiway L near the Runway 25 hold line at the threshold. The traffic was cleared to taxi into position on Runway 25 while the ownship was on approach to Runway 34R. When the ownship crossed the threshold, the traffic began its departure on Runway 25 without receiving clearance.

\section{Scenario 4-departure/departure}

Scenario 4 was another crossing runway scenario where two aircraft were departing simultaneously on crossing runways. The ownship was initially on Taxiway C approximately 500' from the Runway 34R threshold traveling at $8 \mathrm{kts}$. The traffic was initially on Taxiway L near the Runway 25 hold line at the threshold. The traffic was cleared into position on Runway 25 while the ownship taxied on C. The EP was cleared for departure on Runway 34R. Once the ownship began its departure (on runway heading and traveling greater than $10 \mathrm{kts})$, the traffic began its departure without receiving clearance.

\section{Scenario 5 - taxi crossing/departure}

Scenario 5 was designed to evaluate the taxi crossing situation. The ownship began on the ramp at the Mercury Air Center facing Taxiway L. The traffic began on Taxiway $L$ at the Runway 25 hold line at the runway threshold. The traffic was cleared to taxi into position while the ownship taxied out of the ramp. The ownship was cleared to taxi to Runway 34R, with instructions to cross Runway 25 (without stopping). When the ownship crossed the centerline of Taxiway L, the traffic began its departure without receiving clearance.

\section{Evaluation Pilots}

Sixteen GA pilots served as participants in the experiment with an equal distribution of flying 
experience used to represent a cross-section of the Part 91 pilot population: low-time ( $<400$ hours) visual flight rules (VFR), high-time ( $>400$ hours) VFR, low-time $(<1000$ hours) instrument-rated, and high-time ( $>2000$ hours) instrument-rated.

\section{Test Matrix}

The testing was conducted in two phases. A "rare event" study evaluated pilot reaction to a runway incursion event with a given display condition and incursion detection algorithm without expectation on the part of the subject as to the true intent of the study. Four display conditions (BMO, BMOT, BAMO, BAMOT) were evaluated across subjects (each EP was given only one display condition). Eighteen runs were randomly flown that consisted of six different approaches in varying day time visual meteorological conditions (VMC) and instrument meteorological conditions (IMC) visibility conditions. The intent of these runs was to provide sufficient variety and task demands to hide the true focus of the study. The final run in the block was the runway incursion event (Scenario 1 above) conducted in VMC conditions ( $3 \mathrm{~nm}$ visibility and 1000' ceiling). Scenario $1-$ the arrival/take-off hold incursion - was selected because of its prevalence in runway incursion incident and accident statistics. A wind profile was introduced to add workload to the tasks.

Following the rare event study phase, a usability study evaluated the display concepts effectiveness for runway incursion prevention. Each EP evaluated all five incursion scenarios with the seven display conditions. The first run for each scenario group used the baseline display condition. All of the runs that provided alerting used the RSM incursion detection algorithm as the alert source. In this study phase, the subjects had an expectation for the study intent (i.e., runway incursions) but they did not know the scenario before the initial run. Another purpose of the usability study was to evaluate the RSM and PathProx ${ }^{\mathrm{TM}}$ incursion detection algorithms for GA operations. Each EP evaluated both algorithms using all five incursion scenarios and the BRIPS display condition. All runs in the usability study were conducted in VMC conditions (3 nm visibility and 1000' ceiling) without winds.

\section{Procedure}

Each EP participated in an extensive briefing and training session prior to data collection that was principally designed to mask the focus of the experiment (runway incursion prevention) for the rare event testing. The EP was trained on the incursion alerting system prior to the rare event testing only if the display condition evaluated included alerting; otherwise, the training was conducted before the usability study. Before each run, the pilots were briefed on the run conditions, e.g. approach or departure, visibility, alerting system selected (for usability study), and displays available. Post-run, post-block, and post-test questionnaires were administered when required. Audio, video, and digital data were also recorded.

\section{Results}

A summary of quantitative and qualitative results is presented for the rare event testing and usability study. All data is referenced from the center of gravity of the aircraft.

\section{Rare Event Testing}

As described above, each EP received 18 various approach tasks before being presented with the runway incursion event (Scenario 1).

The FAA runway incursion severity rating [1], described below, was used to categorize the runway incursion incident data from this study.

- Category A-Separation decreases, extreme action taken to narrowly avoid collision, or collision occurs;

- Category B - Separation decreases, significant potential for collision;

- Category C-Separation decreases, ample time and distance to avoid collision;

- Category D - Little or no chance of collision but meets definition of runway incursion.

Fourteen scenarios resulted in the less hazardous Category $\mathrm{C}$ and $\mathrm{D}$ incursions, one resulted in a Category A incursion, and one resulted in a Category $\mathrm{B}$ incursion.

The 14 less hazardous Category C and D incursions were mitigated by the EPs by conducting a go-around and gaining separation from the traffic. 
The Category A runway incursion occurred with the EP flying the BMOT display concept. Despite the traffic indications on the surface map and out-the-window visuals, the EP demonstrated no awareness of the runway traffic and over-flew the traffic and landed.

The Category B incident occurred when the EP over-flew the runway traffic (at 146' AFL) before conducting a go-around. The EP was aware of the incursion after having received an audible alert (BAMO display concept) but continued to descend to visually acquire the traffic to confirm the alert. This incident would have been classified as a Category D incursion if the EP had initiated the goaround at first awareness of the alert.

No statistically significant differences were found between the display concepts for the distance to the incurring traffic when a go-around was initiated by the pilots or for the EP's reaction time from the incursion event occurrence (based on a Multivariate Analysis of Variance (MANOVA) test $\mathrm{p}>0.05)$.

Data on both incursion detection algorithms were collected during the incursion events; however, only the alerts generated by the RSM algorithm were displayed to the EP, when the display condition included alerting. For all incursion runs, the RSM issued an alert when the ownship was an average distance of 4060' and approximately 24 seconds from the traffic. PathProx ${ }^{\mathrm{TM}}$ computed that a Caution alert was necessary when ownship was 5836' and approximately 35 seconds from the traffic and a Warning alert was necessary when ownship was 4234' and approximately 25 seconds from the traffic. (Data from two EPs were omitted from these averages because the pilots were initially heading to the wrong runway and turned toward Runway 34R within $1.1 \mathrm{~nm}$. Even though alerts were generated the results were skewed.) A MANOVA showed a significant effect between the incursion detection algorithms tested $(\mathrm{F}(4,42)=$ $190.163, \underline{p}<.0001)$. A subsequent ANOVA on the dependent variables revealed that the PathProx ${ }^{\mathrm{TM}}$ Caution alert was generated significantly earlier $(\mathrm{F}(2,42)=6.839)$ and at a greater distance from the incursion aircraft $(F(2,42)=7.302)$ than from either the PathProx ${ }^{\mathrm{TM}}$ Warning alert or the RSM Warning alert. There were no significant differences between the PathProx ${ }^{\mathrm{TM}}$ Warning alert and RSM Warning alert.

Of the 16 runs, eight EPs initially became aware of the incursion traffic before the alert would have occurred by viewing out the window; five visually acquired the traffic out the window after an incursion alert, if used, would have occurred; one saw the traffic on the surface map well before the alert occurred; and two EPs did not see the traffic at all (the category A and B incursions described above). As shown in Figure 6, the incursion traffic was acquired sooner when the EP was provided with a traffic display on the surface map and/or incursion alerts, but the differences were not statistically significant at the $\alpha=0.05$ level. The data for the two EPs that did not acquire the traffic and the two EPs that headed to the incorrect runway were omitted from Figure 6.

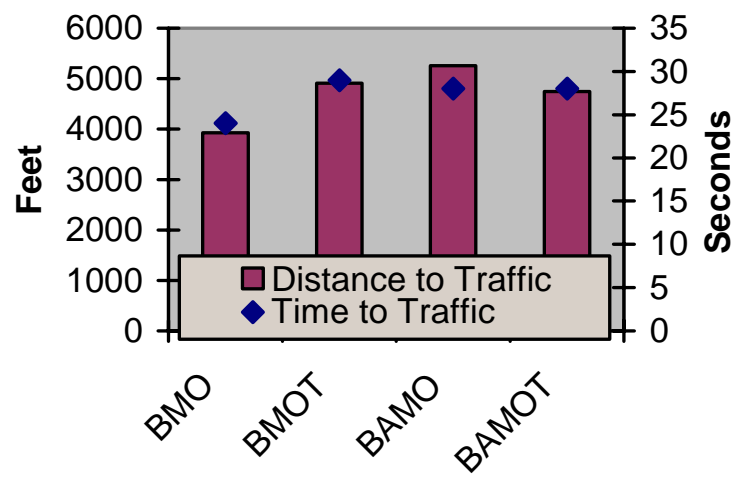

Figure 6. Initial Traffic Awareness

For those displays that had alerting (BAMO, BAMOT), there were no significant differences in timeliness of the alerting in terms of being able to take evasive action. However, when pilots were asked to rate all four display concepts on the perceived efficacy of the alerts $(\mathrm{F}(3,16)=10.948)$ and the additional safety value added $(\mathrm{F}(3,16)=$ 8.814) an ANOVA revealed a significant effect between the displays $(\mathrm{p}<0.01)$. Subsequent posthoc Student Newman Kuels tests showed that pilots reported that the BMO display condition was significantly poorer than the other three display conditions which were not significantly different from each other. 


\section{Usability Study}

All test runs conducted during the usability study included incursion events. During each test run, data were simultaneously collected on the performance of both incursion detection algorithms; however, only one method was chosen for display.

The EPs evaluated all five incursion scenarios with the seven display conditions to determine the effectiveness of the display for runway incursion prevention. The RSM was used as the alert source for this testing phase. Although the EPs were aware that each run would contain an incursion event, they were not told the type of incursion before the initial run for each scenario. The initial run evaluated the baseline display condition. This was done to determine if the EP would visually acquire the incursion traffic out the window since the surface map and alerting were not available.

The EPs also evaluated the RSM and PathProx ${ }^{\mathrm{TM}}$ incursion detection algorithms for GA operations using the five incursion scenarios and the BRIPS display condition.

\section{Quantitative Results}

A total of 612 test runs were completed. Since the $\mathrm{BMO}$ and $\mathrm{BAMO}$ display conditions provided similar alerting information to the $\mathrm{B}$ and $\mathrm{BA}$ display conditions, a limited number of BMO and BAMO test runs were conducted in the interest of time. Data was not analyzed for 16 runs due to missing data files, yielding a total of 596 runs. Alerts were required for display on 432 test runs. During these 432 runs, the RSM was chosen as the alert source 80 percent of the time (347 runs).

RSM generated alerts on 533 runs. RSM did not alert on 60 runs due to the maneuver taken by the EP. For example, the EP may have acquired the traffic out the window and conducted a go-around before the alerting criteria were met. RSM did not alert on three runs due to the scenario timing (no incursion event). For the PathProx ${ }^{\mathrm{TM}}$ algorithm, Caution alerts were only possible on 243 runs and of these, alerts were generated on 157 runs. Of the possible 596 test runs, PathProx ${ }^{\mathrm{TM}}$ generated warning alerts on 352 runs. The 244 runs in which PathProx ${ }^{\mathrm{TM}}$ did not alert were generally due to the maneuvering performed by the EP. For instance, when RSM alerting was provided, the pilot executed an aircraft maneuver and this generally occurred before the PathProx ${ }^{\mathrm{TM}}$ alerts were generated. Detailed PathProx ${ }^{\mathrm{TM}}$ analysis was not possible because specific alerting criteria is proprietary.

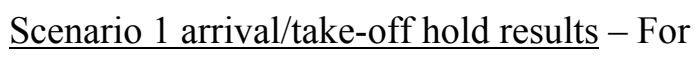
the Baseline display (B) condition, all EPs acquired the incursion traffic out the window for Scenario 1 when approximately $1 \mathrm{~nm}$ from the runway threshold. As a result, the average go-around initiation point was 443' AFL and 5240' (approximately 28 seconds) from the traffic. The EP initiated a go-around after the PathProx ${ }^{\mathrm{TM}}$ Caution alert would have occurred on average (475, AFL and 5641' - approximately 34 seconds - from the traffic) but before either the RSM (409' AFL and 4272' - approximately 24 seconds - from the traffic) or PathProx ${ }^{\text {TM }}$ (392' AFL and 4117' approximately 25 seconds - from the traffic) Warning alert would have occurred.

Post-run data analysis on all Scenario 1 test runs during the usability study showed that the PathProx ${ }^{\mathrm{TM}}$ Caution alert was generated when the ownship was at approximately 608' AFL and 5756' (approximately 36 seconds) from the incurring traffic. Both the RSM (488' AFL and 4121' approximately 25 seconds - to traffic) and PathProx ${ }^{\text {TM }}$ (492' AFL and 4173' - approximately 25 seconds - to traffic) Warning alerts were generated at essentially the same moment. It should be noted that during the test runs in which alerts were provided, the EP was asked to continue the maneuver until the alert was received for evaluation purposes.

Scenario 2 departure/intersection departure results - Six EPs (37.5 percent) did not acquire the incursion traffic visually for Scenario 2 or saw the traffic too late to abort the departure and actually over-flew the runway traffic when using the Baseline (B) display condition.

Figure 7 shows when the EP began to abort the departure to avoid a conflict with the incursion traffic. A greater safety margin resulted when alerts were provided (BA, BAMO, BAMOT, and BRIPS). The EP also aborted the departure for all of these runs. The departure was aborted later when alerting was not provided (B, BMO, and BMOT), although still with enough time to stop prior to reaching the traffic. Also, as discussed above, the EP actually 
took off on six runs and over-flew the traffic, using the Baseline display.

For this departure scenario, the RSM Warning alert (ownship at $28 \mathrm{kts}$ ground speed and 2638' approximately 21 seconds - from Taxiway L) was generated before and at a lower ground speed than the PathProx ${ }^{\mathrm{TM}}$ Warning alert (41 kts ground speed and 2452' - approximately 19 seconds - from Taxiway L); however, both provided ample time to abort and stop before reaching the incurring traffic. PathProx ${ }^{\mathrm{TM}}$ Caution alerts are not generated on departure.

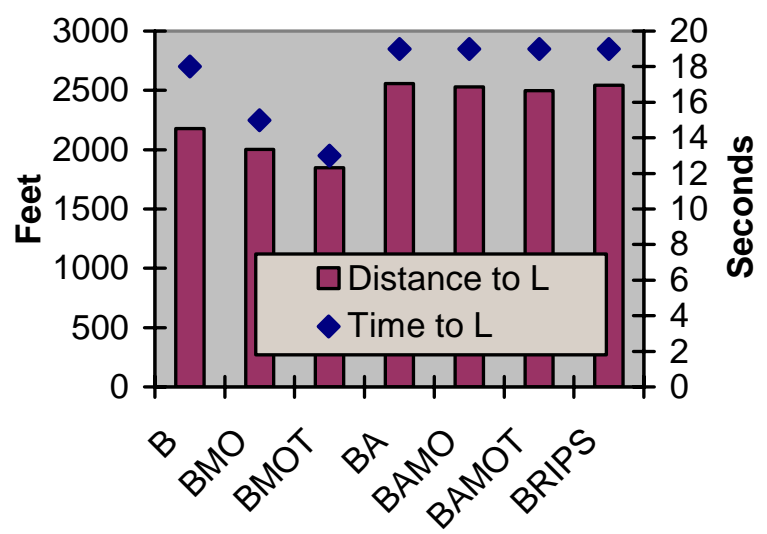

Figure 7. Scenario 2 Abort Initiation

Scenario 3 arrival/departure results - For the Baseline (B) condition, 13 EPs (81 percent) did not acquire the incursion traffic visually for Scenario 3. Due to the scenario design; however, 14 EPs landed and stopped before reaching the crossing runway. One EP landed but taxied through the intersection as the traffic was departing from crossing Runway 25. One EP conducted a go-around.

On average, the RSM Warning alert (1303' approximately 11 seconds - to Runway 25) was generated slightly before the PathProx ${ }^{\mathrm{TM}}$ Warning alert (1181' - approximately 9 seconds - to Runway 25). Both algorithms alerted at or slightly before touchdown. PathProx ${ }^{\mathrm{TM}}$ Caution alerts were not generated for this scenario.

For all Scenario 3 runs (124 total), a go-around was conducted just before touchdown ( 7 , AFL) on only 11 percent (14) of the runs. $\underline{\text { Scenario } 4 \text { departure/departure results - Five }}$ EPs (31.3 percent) saw the incursion traffic visually and aborted the departure when using the Baseline (B) display condition. Nine EPs (62.5 percent) did not see the incursion traffic visually or saw the traffic too late to abort the departure. For these runs, the ownship came within an average distance of 458' (range from 179' to 795') from the incursion traffic. The timing was early for two of the runs resulting in no incursion event; therefore, these data are not included in the analysis.

Generally, a greater safety margin resulted when alerts were provided (BA, BAMO, BAMOT, and BRIPS) as shown in Figure 8. For runs in which the EP acquired traffic, the departure was aborted later when alerting was not provided (B and BMOT); however, with still enough time to stop before the crossing runway.

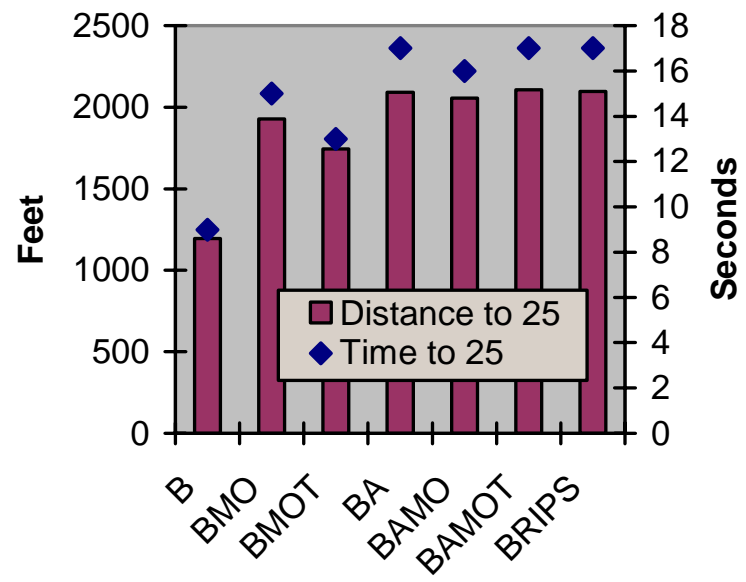

Figure 8. Scenario 4 Abort Initiation

The RSM Warning alerts were generated before the PathProx ${ }^{\mathrm{TM}}$ Warning alerts for this departure scenario. When RSM alerts were presented, the EP typically reacted based on those alerts; therefore, PathProx ${ }^{\mathrm{TM}}$ alerts were sometimes not generated or generated after the abort maneuver. For an accurate assessment of PathProx ${ }^{\mathrm{TM}}$ performance, only the results from the runs in which the PathProx ${ }^{\mathrm{TM}}$ alerts were shown to the EP will be presented. For these runs, the PathProx ${ }^{\mathrm{TM}}$ Warning alerts were generated when the ownship was going $48 \mathrm{kts}$ ground speed and was 1891' and approximately 15 seconds from Runway 25 . The RSM Warning alerts were generated when the 
ownship was going 29 kts ground speed and was 2216' and approximately 18 seconds from Runway 25. Again, PathProx ${ }^{\mathrm{TM}}$ Caution alerts are not generated on departure.

Scenario 5 taxi crossing/departure results Fourteen EPs acquired the incursion traffic out the window when using the Baseline (B) condition and stopped before reaching Runway 25. One EP did not see the traffic and actually crossed Runway 25 in front of the departing traffic. One EP saw the departing traffic out the window but chose to cross Runway 25 anyway.

The RSM algorithm is designed to provide early warning of an impending incursion during taxi, based on the aircraft taxi speed. If the ownship is traveling $8 \mathrm{kts}$ or greater and is not slowing down, the alert will be generated before the aircraft reaches the hold line, providing sufficient distance to stop before crossing the hold line. As the taxi speed increases, the alert is generated when the ownship is a farther distance from the hold line. The alert is not generated until after the ownship crosses the hold line when the ownship is traveling less than $8 \mathrm{kts}$. The $8 \mathrm{kt}$ threshold was used to prevent false or nuisance alerts as the ownship taxis toward a hold line. (As stated earlier, details on the PathProx ${ }^{\mathrm{TM}}$ implementation are proprietary.)

As with scenario 4, the RSM alerts were generated before the PathProx ${ }^{\mathrm{TM}}$ alerts for this scenario. Therefore, for an accurate assessment of PathProx ${ }^{\mathrm{TM}}$, only the results from runs in which the PathProx ${ }^{\mathrm{TM}}$ alerts were given to the EP will be presented. For these runs, the RSM Warning alerts were generated when the ownship was an average distance of 27' before the hold line and 197' from the edge of Runway 25. Table 1 shows that the RSM alert was generated before reaching the hold line (negative value) when the taxi speed was greater than $8 \mathrm{kts}$. A positive value indicates the aircraft crossed over the hold line. The RSM alerting is proactive in preventing an incursion (crossing the hold line) in this situation.

PathProx ${ }^{\mathrm{TM}}$ Caution alerts were generated when the ownship was an average of 18' past the hold line and 152 ' from the runway edge. PathProx ${ }^{\mathrm{TM}}$ Warning alerts were generated when the ownship was 41 ' past the hold line and 129' from the runway edge.
Table 1. Scenario 5 RSM Alert Generation

\begin{tabular}{|c|c|c|}
\hline EP & $\begin{array}{c}\text { Ground } \\
\text { Speed (kts) }\end{array}$ & $\begin{array}{c}\text { Distance to } \\
\text { hold line (feet) }\end{array}$ \\
\hline 1 & 10.7 & -86 \\
\hline 1 & 9.7 & -59 \\
\hline 2 & 9.7 & -48 \\
\hline 3 & 9.2 & -59 \\
\hline 4 & 7.9 & 12 \\
\hline 5 & 7 & 6 \\
\hline 6 & 8.4 & -56 \\
\hline 7 & 6 & 7 \\
\hline 8 & 13.4 & -17 \\
\hline 9 & 10.3 & -70 \\
\hline 10 & 8.4 & -48 \\
\hline 11 & 8.3 & -48 \\
\hline 12 & 7.5 & 17 \\
\hline 13 & 7.4 & 14 \\
\hline 15 & 6.5 & 7 \\
\hline 16 & 10.6 & -8 \\
\hline
\end{tabular}

\section{Qualitative}

Post-run, post-block, and post-test questionnaires were administered when required. Ratings for most of the questions were given on a scale of 1 (low) to 10 (high).

When asked to rate the effectiveness of the perspective surface map compared to the plan view map for prevention of runway incursions, the perspective map was rated slightly more effective (2.9). However, the location of the surface map was suboptimal and should be positioned closer to the pilots head-up field-of-view. The traffic presentation on the surface map was considered easily discernable (8.4). Most EPs (14 of 16) considered traffic presentation necessary to prevent runway incursions. The addition of traffic would provide increased (8.6) situation awareness (SA) over a surface map with only ownship location. Over half of the EPs (10 of 16) indicated graphical presentation of alerts on the surface map was necessary to prevent runway incursions. The addition of graphical alerts would provide increased (6.3) SA. The EPs considered the terms used for the incursion alerts (e.g. "Warning, Traffic Departing 25") to be very effective (8.9 average rating for all phrases).

For display conditions with alerting available, the EPs indicated that the incursion event would 
most likely be brought to their attention first through audible alerting, then on the surface map, and lastly out the window. Nine of 16 EPs indicated that an audible alert alone would provide a minimally effective incursion prevention display, while five of 16 indicated a surface map with ownship and traffic would be minimally effective. However, all 16 EPs indicated a surface map with ownship and traffic in conjunction with an audible alert would be an optimal incursion prevention display. Table 2 shows, according to averaged EP ratings, that for all alerting display conditions, the caution and warning alerting system provided the greatest amount of runway incursion awareness. An ANOVA confirmed this conclusion with significant main effects $(\mathrm{p}<0.0001)$ for display on pilot ratings of situation awareness of where they were located $(F(6,90)=143.956)$ and where other traffic and hazards were located $(\mathrm{F}(6,90)=94.899)$.

Table 2. Alerting Preference for Incursion Awareness and Resulting SA Improvement

\begin{tabular}{|l|c|c|c|c|}
\hline \multirow{2}{*}{$\begin{array}{c}\text { Alerting } \\
\text { Type }\end{array}$} & \multicolumn{2}{|c|}{ Warning only } & \multicolumn{2}{c|}{ Caution \& Warning } \\
\cline { 2 - 5 } & \# of EP & SA & \# of EP & SA \\
\hline BA & 6 & 6.5 & 10 & 4.2 \\
\hline BAMO & 6 & 6.7 & 10 & 4.3 \\
\hline BAMOT & 7 & 7.7 & 9 & 5 \\
\hline BRIPS & 7 & 7.6 & 9 & 5.2 \\
\hline
\end{tabular}

All EPs indicated that both the RSM and PathProx ${ }^{\mathrm{TM}}$ alerting provided sufficient time to avoid a potential incursion conflict. Only six of 16 EPs thought providing both caution and warning alerts was more effective in preventing runway incidents than a single warning alert. However, nine of 16 EPs indicated that the caution and warning system provided greater (4.4) situation awareness and provided more reaction time (even though this was not always the case). Eleven of 16 EPs liked the idea of having a caution alert in conjunction with a warning to provide more evaluation and reaction time, i.e. a greater comfort level. For the scenarios evaluated, the EPs generally felt that providing caution and warning alerts on approach was most effective, while a warning alert alone was sufficient when on the airport surface (during departure and taxi). This was particularly true for Scenario 5. Many EPs indicated that the short caution alert had no benefit.

The majority of EPs would like to be provided with maneuver guidance for conflict resolution in addition to runway incursion alerting on final approach (12 of 16) and when taxiing across a runway (nine of 16). Half of the EPs would like maneuver guidance on departure.

In general, the EPs felt safer during runway incursion incidents when alerting was provided $(\mathrm{B}=2.1, \mathrm{BMO}=2.75, \mathrm{BMOT}=5.4, \mathrm{BA}=7.2$, $\mathrm{BAMO}=7.4$, $\mathrm{BAMOT}=9.3$, and BRIPS $=9.2$ ). Analysis of pilot responses to their perceived safety and runway incursion prevention effectiveness support this conclusion with significant main effects found (i.e. for perceived safety $(F(6,90)=857.390)$ and runway incursion prevention effectiveness $(\mathrm{F}(6$, $90)=188.793)$ ). The addition of traffic was marginally beneficial when presented on a moving map display and was only effective when alerting was provided. A possible cause may be that pilots had to transition to out-the-window and were not focused on the head-down display. With alerting, the pilot is provided a cue to direct focus and attention to the head-down display to locate the incurring traffic. In fact, pilots rated having audible alerts (BA) and having alerts with a map with ownship but no traffic (BAMO) similarly for runway incursion prevention on almost all dependent variables measured. For the experimental scenarios tested, the moving map display revealed its utility only when traffic AND alerting were options.

\section{Summary}

A Runway Incursion Prevention System (RIPS) adapted for general aviation operations was evaluated in a simulation study in the fall of 2005. The purpose of the study was to evaluate the airborne incursion detection algorithms and associated alerting and airport surface display concepts for general aviation operations using a rare event study followed by a usability study.

The results indicate that, during the rare event testing, most pilots were able to acquire the incurring traffic looking out the cockpit windows (in VMC conditions), even before incursion alerting 
was activated. In the usability study, varying outthe-window detection of incurring traffic was noted and the importance of incursion detection algorithms was shown.

The surface map with ownship (without other traffic) was rated as being significantly inferior to a surface map with traffic and/or incursion alerting for perceived safety value added. However, one pilot experienced a severe runway incursion risk for collision despite having traffic displayed on a surface map. The addition of traffic was marginally beneficial when presented on a surface map display and was only effective when alerting was provided. A possible cause may be that pilots had transitioned to out-the-window and were not focused on the head-down display to locate the incurring traffic. Pilots reported that the utility of the surface map would be significantly more effective if located higher on the instrument panel closer to the pilot's head-up field-of-view.

The results generally matches past research on commercial operations that the incursion alerts provided sufficient time to avoid a potential incursion conflict. Departures were generally aborted sooner when alerts were provided, resulting in greater safety margins. A surface map with ownship and traffic along with audible alerts was considered an optimal incursion prevention display, while an audible alert alone was considered a minimally effective display. Over half of the pilots would like maneuver guidance for conflict resolution in conjunction with incursion alerting. In general, the pilots felt substantially safer during runway incursion incidents with alerting onboard.
Demonstration and Testing at the Dallas/Fort Worth International Airport, Proceedings of the $20^{\text {th }}$ Digital Avionics Systems Conference.

[5] Jones, Denise R., 2002, Runway Incursion Prevention System Simulation Evaluation, Proceedings of the AIAA/IEEE $21^{\text {st }}$ Digital Avionics Systems Conference.

[6] Jones, Denise R., 2005, Runway Incursion Prevention System Testing at the Wallops Flight Facility, Proceedings of the SPIE Defense \& Security Symposium.

[7] Bartolone, Anthony P., L. J. Glaab, M. F. Hughes, R. V. Parrish, 2005, Initial Development of a Metric to Describe the Level of Safety Associated with Piloting an Aircraft with Synthetic Vision Systems (SVS) Displays, Proceedings of the SPIE Defense \& Security Symposium.

[8] RTCA SC-193, 2001, User Requirements for Aerodrome Mapping Information, RTCA/DO-272.

[9] Green, David F., February 2006, Runway Safety Monitor Algorithm for Single and Crossing Runway Incursion Detection and Alerting, NASA CR-2006214275.

[10] Cassell, Rick, C. Evers, J. Esche, 2003, Safety Benefits of PathProx - A Runway Incursion Alerting System, Proceedings of the AIAA/IEEE $22^{\text {nd }}$ Digital Avionics Systems Conference.

\author{
$25^{\text {th }}$ Digital Avionics Systems Conference \\ October 15, 2006
}

\section{References}

[1] FAA Office of Runway Safety, August 2005, FAA Runway Safety Report.

[2] National Transportation Safety Board, November 2005, Most Wanted Transportation Safety Improvements, www.ntsb.gov/recs/mostwanted/index.htm

[3] National Transportation Safety Board, July 2000, Safety Recommendation, Letter to the FAA Administrator, A-00-66.

[4] Jones, Denise R., C. C. Quach, S. D. Young, 2001, Runway Incursion Prevention System - 